\title{
Microscope harmonies
}

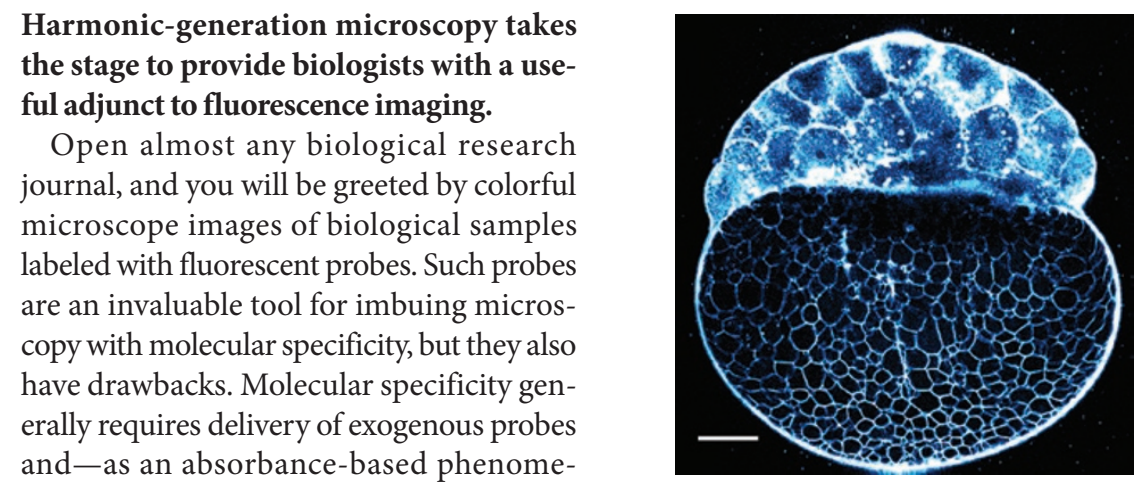
non-fluorescence is plagued by dyebleaching, saturation, blinking and, as highlighted on page 782 , phototoxicity.

Under the right circumstances, certain molecules and structures can mediate a nonlinear harmonic optical process in which two or more low-energy photons combine to form a single higher-energy photon. These harmonic processes are not mediated by absorbance and thus avoid many concerns with fluorescent dyes.

This sounds great; so why is harmonicgeneration microscopy still largely offstage? For one thing, harmonic-generation microscopy typically uses expensive femtosecond lasers. Second, light from the sample is emitted mostly in the forward direction-complicating detection. Finally, few compelling biological applications have been reported. Two reports suggest biologists should give this technology a more serious look.

The first, by Emmanuel Beaurepaire, Nadine Peyriéras and colleagues in France and Spain, describes label-free imaging of early development in zebrafish using harmonic-generation microscopy (Olivier et al., 2010). Fluorescence imaging of very early development can be difficult owing to insufficient time for expression, maturation and proper localization of fluorescent protein probes used to identify individual cells and subcellular structures. Beaurepaire and colleagues found that third-harmonic generation (THG) imaging-mediated by three combining photons-highlighted cell
Raw single-plane THG image of an unstained zebrafish embryo in sagittal orientation. Scale bar, $100 \mu \mathrm{m}$. Image courtesy of Emmanuel Beaurepaire.

boundaries better than a membrane-targeted fluorescent protein, and an increasing signal served as a valuable control for photodamage. Beaurepaire says, "Typically the contrast of the fluorescent image isn't as good as these third-harmonic images."

Furthermore, the second-harmonic generation (SHG) signal-produced by two combining photons-from mitotic spindles in dividing cells allowed accurate determination of division time. "[The SHG signal] is very small and difficult to catch. You need optimized microscopes and intelligent scanning," says Beaurepaire. They developed a spiral-shaped scanning strategy that delivers most of the energy to the center of the embryo, where the signal is weakest, and rapidly scans the periphery. This optimized the acquisition time and minimized the amount of light sent to the sample for a given signal level, limiting photodamage.

Combining the cell-division information from SHG signals with the cell-boundary information from THG signals aided in reconstructing cell lineages through the first ten cycles of cell division. The resulting data provided a detailed view of the earliest stages of development and highlighted a lengthening of the cell cycle in individual cells with unprecedented precision.

Although the ability to image unlabeled samples is a compelling capability of harmonic-generation microscopy and the technique may limit some photodamage pathways, it requires high illumination intensities. Periklis Pantazis from Scott Fraser's lab at the California Institute of Technology remarks, "Beaurepaire's paper is beautiful, but it works best at earlier stages of development and can't selectively label individual cells so that they can be followed over time." Pantazis's goal was to find bright, photostable, $\mathrm{pH}$-insensitive and color-selective SHG probes that could be imaged at low power by biologists with conventional microscopes. Many nanocrystals have been reported that generate SHG signals, but Pantazis wanted to evaluate their performance for in vivo imaging. $\mathrm{He}$ says, "We tested them primarily in zebrafish, because you can efficiently collect the forward-directed SHG signal from endogenous structures for signal comparison."

Pantazis and colleagues found that tetragonal barium titanate performed best, and was easier to excite and to detect than endogenous structures (Pantazis et al., 2010). These nanoprobes had strong forward- and backward-directed signals, allowing standard epi-detection. Unlike quantum dots, the SHG nanoprobes did not blink, bleach or saturate during excitation, and they had a narrower signal profile. In preliminary experiments in mice, no toxicity was evident, although the nanoprobes accumulated in the spleen, kidney and other locations. Functionalization is more straightforward than with quantum dots, and the probes do not need to be coated for cell compatibility.

Harmonic-generation microscopy will not replace fluorescence microscopy but these reports show that it is a powerful complement. Daniel Evanko

\section{RESEARCH PAPERS}

Olivier, N. et al. Cell lineage reconstruction of early zebrafish embryos using label-free nonlinear microscopy. Science 329, 967-971 (2010).

Pantazis, P. et al. Second harmonic generating (SHG) nanoprobes for in vivo imaging. Proc. Natl. Acad. Sci. USA 107, 14535-14540 (2010). 